\title{
Use of transoral CO2 laser microsurgery for treatment of cancerous neoplasms of the larynx in Vietnam
}

L.M. Ky ${ }^{1}$, P.V. Huu ${ }^{3}$, N.X. Quang ${ }^{3}$, T. Hai ${ }^{5}$, M.Q. Hoan ${ }^{4}$, N. BacHai ${ }^{2}$ and N.D. Phuc ${ }^{2}$

${ }^{1}$ National Otorhinolaryngology Hospital of Vietnam, Vietnam National University, Hanoi (VNU)

${ }^{2}$ Hanoi Medical University, Vietnam

${ }^{3}$ National Otorhinolaryngology Hospital of Vietnam

${ }^{4}$ Tay Nguyen University, Daklak, Vietnam

${ }^{5}$ Nghe An Maternity - Pediatric Hospital, Viet Nam

Corresponding author: N.D. Phuc

E-mail: Phucnguyendinh11@yahoo.com.vn

Genet. Mol. Res. 20 (1): gmr18734

Received December 30, 2019

Accepted April 20, 2020

Final Revision January 25, 2021

Published March 25, 2021

DOI http://dx.doi.org/10.4238/gmr18734

ABSTRACT. Laryngeal cancer remains one of the most common head and neck tumors. In the treatment of this disease, it is important to assess the overall survival when using transoral $\mathrm{CO}_{2}$ laser microsurgery. The aim of the research was to make an evaluation of treatment of patients with laryngeal cancer undergoing $\mathrm{CO}_{2}$ laser transoral microsurgery. Fifty patients aged $40-82$ years (average age 58.7 years) with early-stage laryngeal cancer were treated with $\mathrm{CO}_{2}$ laser transoral microsurgery at the National ENT hospital from January 2012 to August 2014.Tumors at the T1aN0M0 stage were detected in $68 \%$, T1bN0M0 in $14 \%$, T2N0M0 in $18 \%$. Overall survival was $87.1 \pm 2.3$ months, and 5 -year survival was $92 \%$. The survival rate depending on the type of surgical intervention Type III, Type IV, Type Va, Type Vb, Type Vc was 93, 94, 100, 100, and $100 \%$, respectively. The survival rate depending on the stage T1aN0M0 was $91 \%$, on the stage T1bN0M0 was $100 \%$, on the stage T2N0M0 was $88 \%$. Transoral $\mathrm{CO}_{2}$ laser microsurgery shows 
excellent results in laryngeal tumors, especially in the T1aN0M0 stage, making it possible to perform surgery in more comfortable conditions; it provides high survival rates, excellent cosmetic and functional results compared to classical open access, without compromising immediate and long-term treatment results.

Key words: $\mathrm{CO}_{2}$ transoral laser microsurgery; Larynx; Carcinoma; T1- T2; Survival

\section{INTRODUCTION}

Laryngeal cancer remains one of the most common head and neck tumors, and although significant progress has been made over the past few decades, the diagnosis and treatment of laryngeal cancer, given its complexity, is a unique problem (Le et al., 2019). In particular, squamous cell carcinoma of the larynx accounts for $30-50 \%$ of all neoplasms in the head and neck: 157,000 new cases were diagnosed worldwide in 2012 (Ferlay et al., 2015). As a result of the evolution of diagnostic measures, treatment strategies, and followup protocols, early- stage treatment of laryngeal cancer (T1-T2) has transformed from classical methods of open surgical resection into a less aggressive and more functional endoscopic approach over the past 40 years. Today, the efficiency and favorable outcome of transoral resection using a $\mathrm{CO}_{2}$ laser for pharyngeal cancer (T1-T2) are 80-90\% (Mendelsohn and Remacle, 2018). The main advantages of transoral laser microsurgery (TLM) are the ability to minimize damage to the larynx, maximize the preservation of healthy tissues, as well as respiratory, chewing, and speech functions (Chiesa-Estomba et al., 2019). In addition, the benefits of this type of treatment include a reduced risk of fistula, abscesses, and osteoradionecrosis, as well as a shorter hospital stay, which significantly reduces treatment costs (Golusiński et al., 2019). A number of large studies published between 2000 and 2010 showed that TLM shows excellent results with very high diseasespecific survival for all stages of early carcinoma, especially with Tis and T1 (Sjögren, 2017).

Previous studies have found that postoperative local outcomes, vocal function preservation, survival, and long-term prognosis are comparable to patients who received transoral laser excision therapy, open laryngectomy, and radiation therapy (Mendenhall W., 2004). Since the oncological outcomes of the described methods were comparable in a number of studies, determining the consequences for the quality of life of patients, the cost and duration of treatment should be taken into account in individual cases when determining the method of primary treatment for this category of patients (Park J., 2019).

However, transoral laser microsurgery has several disadvantages. These include certain difficulties in providing hemostasis, access and tissue manipulation (Howard et al., 2016). Inadequate effects on the larynx, damage to the anterior commissure in the craniocaudal plane, invasion of the pre-epiglottic space, massive infiltration of the preepiglottic space, and even slight erosion of the thyroid cartilage impose certain restrictions on the use of TLM (Peretti et al., 2016). In connection with the foregoing, the aim of the study was to evaluate the treatment of patients with laryngeal cancer using $\mathrm{CO}_{2}$ laser transoral microsurgery. 


\section{MATERIAL AND METHODS}

Fifty male patients diagnosed with early-stage laryngeal cancer aged 40-82 years (mean age 58.7 years) were operated at the National ENT Hospital during the period from January 2012 to August 2014 (Figure 1). All patients provided written informed consent to participate in the study, as well as to use the data in this article.

The study protocol was approved by an independent ethics committee. All procedures were carried out in accordance with the ethical principles of the Helsinki Declaration.

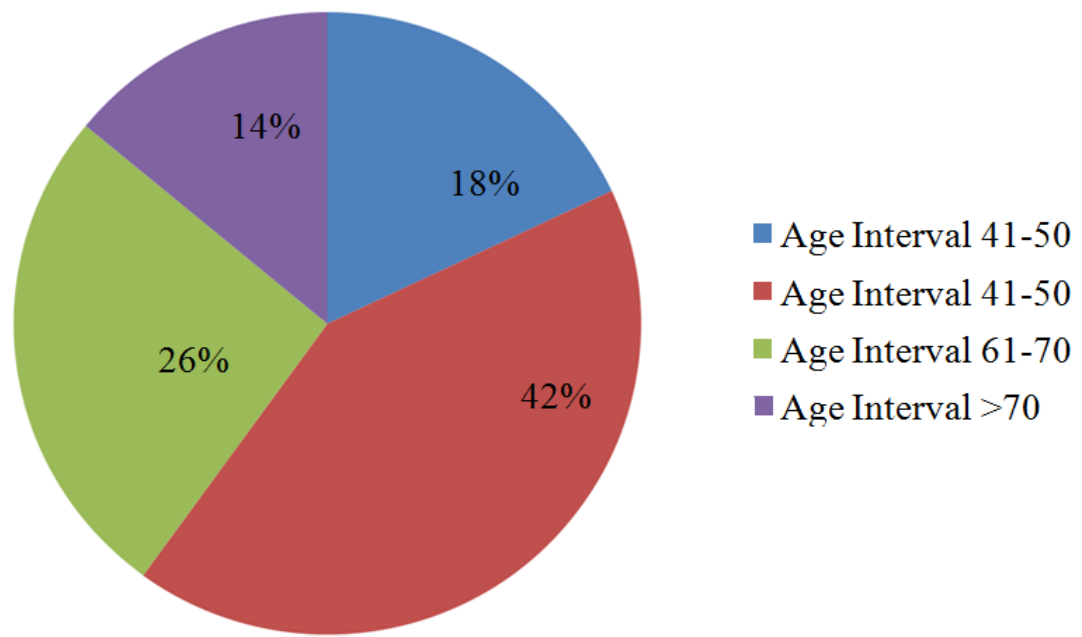

Figure 1. Distribution of study participants who were operated for cancerous neoplasms of the larynx at the National ENT Hospital during the period from January 2012 to August 2014 by age

Among patients, $72 \%$ abused smoking, 36\% abused alcohol, and $40 \%$ abused smoking and alcohol in this cohort. The types of laryngeal lesions that occurred in our study are presented in Table 1. Tumors at the T1aN0M0 stage were detected in 34 cases $(68 \%)$, at the T1bN0M0 stage were detected 7 cases (14\%), at the T2N0M0 stage were detected 9 cases $(18 \%)$.

Table 1. The types of laryngeal lesions that were revealed in the study.

\begin{tabular}{llll}
\hline Lesions & N & \% & \\
\hline 1 & CV, Anterior commissure intact & 25 & 50 \\
1 & CV, Anterior commissure involve & 15 & 30 \\
2 & CV, Anterior commissure involve & 10 & 20 \\
\hline
\end{tabular}

The availability and stage of neoplasm were determined during the preoperative examination using micro laryngoscopy. All patients underwent a biopsy to diagnose tissues, as well as an pan-endoscopy to exclude tumors in the upper digestive tract. Distant metastases were excluded by chest $\mathrm{x}$-ray and abdominal ultrasound. 
Statistical processing. The results were recorded prospectively with subsequent recording and storage in the database. Observation data was available to all patients. The total observation interval was $87.1 \pm 2.3$ months. Survival was calculated by the Kaplan - Meier method. All data were expressed descriptively and compared between subgroups. The data were analyzed using statistical methods (packages SPSS version 22, EZR version 1.38 (Saitama Medical Center, Jichi Medical University.

\section{RESULTS}

Depending on the stage of the tumor, its location and the volume of tissue removed, a different type of surgical intervention was used in accordance with the classification of cordectomies of the European Laryngological Society (ELS) (Table 2).

Table 2. Types of surgical intervention used to remove larynx tumors.

\begin{tabular}{lllllll}
\hline Type & Type III & Type IV & Type Va & Type Vb & Type Vc & Type Vd \\
\hline $\mathrm{N}$ & 13 & 17 & 17 & 2 & 1 & 0 \\
\hline
\end{tabular}

In some cases, postoperative radiation therapy was used (Figure 2).

\section{Post- Op Radiation}

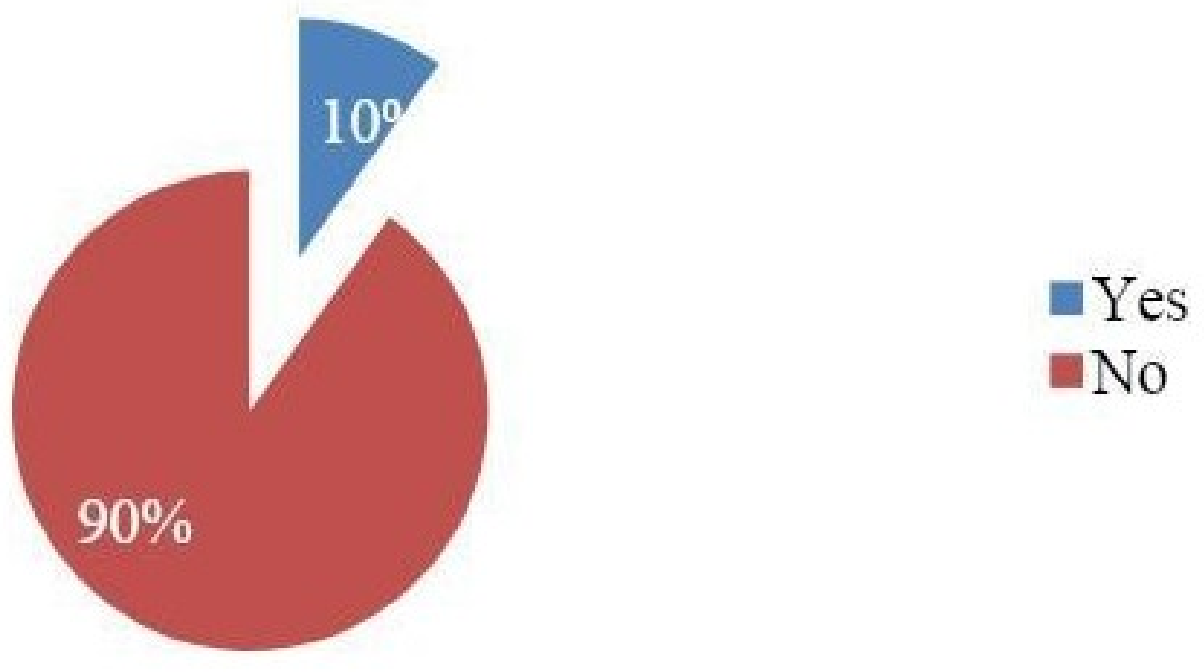

Figure 2. The proportion of postoperative radiation therapy in order to improve the outcome

Survival based on the stage of the tumor process is presented in Table 3. 
Table 3. Survival of operated patients based on larynx tumor stage.

\begin{tabular}{llcl}
\hline Stage & n & $\begin{array}{c}\text { Time of survival } \\
>\mathbf{5} \text { years } \\
\mathbf{\%}\end{array}$ & mean (months) \\
\hline T1aN0M0 & 31 & 91.2 & $68.87 \pm 9.5$ \\
T1bN0M0 & 7 & 100 & $65.13 \pm 5.2$ \\
T2N0M0 & 8 & 87.5 & $64 \pm 3.2$ \\
\hline
\end{tabular}

Survival depending on the type of surgical intervention is shown in Table 4.

Table 4. Survival of operated patients according to operative types of tumors removing.

\begin{tabular}{llll}
\hline OP types & n & $\begin{array}{c}\text { Time of survival } \\
\text { > 5 years } \\
\mathbf{\%}\end{array}$ & mean (months) \\
\hline Type III & 12 & 92.3 & $66.7 \pm 8.4$ \\
Type IV & 15 & 93.8 & $72.8 \pm 10.3$ \\
Type Va & 17 & 94.4 & $63.8 \pm 3.3$ \\
Type Vb & 2 & 50 & $61.0 \pm 1.3$ \\
Type Vc & 1 & 100 & $64.0 \pm 0.8$ \\
\hline
\end{tabular}

In our study, relapse was detected in $8(16 \%)$ patients (Table 5).

Table 5. The number of identified relapses in larynx tumor operated patients during the observation period.

\begin{tabular}{lll}
\hline Relapse - Metastasis & $\mathbf{n}$ & $\mathbf{N}=\mathbf{5 0}$ \\
\hline Local relapse & 2 & $\mathbf{\%}$ \\
Cervical Lymph node metastasis & 0 & 4 \\
\hline
\end{tabular}

\section{DISCUSSION}

In laryngeal cancer, it is important to choose a surgical approach that provides adequate access and visualization (Baskin et al., 2018). The TLM method, especially at stages T1 or T2, shows some of the best results (Mehanna et al., 2016), and application of the $\mathrm{CO}_{2}$ lasers for laser transoral resection of laryngeal cancer is the gold standard (Strieth et al., 2020). In particular, the transoral laser microsurgical technique offers a beneficial alternative to primary chemotherapy (Lane et al., 2020).

Studies comparing the treatment of laryngeal cancer with TLM and radiation therapy give somewhat conflicting results. For example, one study showed that survival was higher in patients with Tis/T1a laryngeal cancer who underwent TLM compared with radiation therapy (Guimarães et al, 2018). In turn, another study showed that the oncological relapse rate is higher in patients with T1a and T1b treated with TML compared to radiation therapy (Gioacchini et al, 2017). The third study did not reveal differences over 
a 5-year period in patients with laryngeal cancer in stage T2 (Warner et al., 2017). According to the latest data in this area (2003-2018), the overall five-year survival rate is $90 \%$, the survival rate for concomitant diseases is $100 \%$, and the larynx retention rate is $85 \%$ (Forner et al., 2020). In another study, the five-year level of local control was: Tis $81.5 \%$, T1a $-88 \%, \mathrm{~T} 1 \mathrm{~b}-100 \%$, and $\mathrm{T} 2-58 \%$; the overall 5 -year survival rate is $87 \%$, and the degree of organ preservation is $93 \%$ (Shiner et al., 2020). In the work of Lei et al. (2019) the local relapse rate for stages Tis, T1a, T1b, and T2 was 25, 22.7, 23.4, and 22.1\%. In our study, a relatively small number of relapses and a high 5-year survival rate are observed, which indicates a significant positive effect of this type of treatment for laryngeal cancer.

Compared with open larynx surgery methods, TLM as a method of treating laryngeal cancer combines high results with a simultaneous reduction in the number of complications, improved voice and swallowing capabilities, and reduced hospitalization (Meulemans et al., 2017). According to the literature, the 5-year survival rate after laser surgery of $\mathrm{T} 1$ carcinomas of the vocal folds is $74-100 \%$, according to other sources, the 5year survival rate after TLM tumors in the T1 / T2 stage is $82 \%$ (Wiegand, 2016). The results of 27-year-old observations (1979-2006) were published, including 404 cases of T1a carcinoma, which reported a local control rate of $86 \%$, laryngeal preservation of $97 \%$ and a survival rate of up to $98 \%$ (Canis et al ., 2015). Chiesa-Estomba et al. (2016) reported local control in $79.3 \%$, with one operation including T1a, T1b and T2 tumors reaching $98.3 \%$ of the local control.

One of the main issues in the treatment of laryngeal cancer is the subsequent quality of life. The work (Vilaseca et al, 2016) showed that $94 \%$ of 401 patients who underwent $\mathrm{CO}_{2}$ TLM had a high quality of life one year after treatment. Another publication demonstrates that voice and swallowing function after this type of treatment remains unchanged over time and does not worsen (Valls et al., 2016).

The involvement of anterior commissure in the cancer process has long been considered a risk factor for relapse. For example, it was found that in patients with Tis-T2 carcinoma of the vocal fold and lesion of the anterior commissure, local control, the degree of larynx preservation, and survival are reduced (Hoffmann et al., 2016). However, it is emphasized that when assessing a tumor in the anterior commissure, it is important to assess the degree of damage in the horizontal and vertical planes affecting different areas of the pharynx, since tumors with a purely horizontal spread are a good indicator for $\mathrm{CO}_{2}$ TLM (Peretti et al., 2016). Studies in Vietnam indicate that the use of $\mathrm{CO}_{2}$ TLM for the treatment of patients with cancer of the oropharyngeal zone shows high positive results (Tran and Nguyen, 2018). In another work involving Vietnamese patients, laser surgery gives a very good positive prognosis for pharyngeal cancer in stages $\mathrm{T} 1$ and $\mathrm{T} 2$ with a low number of complications (Nguyen and Tran, 2017). In our study, in some cases, there was a lesion of the anterior commissure, however, as the results showed, 5-year survival, despite this factor, remained at a fairly high level.

\section{CONCLUSIONS}

The results of the study lead us to suggest that the use of $\mathrm{CO}_{2}$ laser provides thorough hemostasis, making it possible to carry out the operation under more comfortable conditions, allowing to clearly see the border between healthy and tumor tissue and more 
accurately determine the prevalence of tumor processes. A significantly smaller area of coagulation necrosis allows doctors to save a significant amount of healthy tissue without the use of plastic surgery.

Transoral laser microsurgery in the treatment of laryngeal cancer (T1-T2) provides high survival rates and excellent cosmetic and functional results compared to classic open access, without compromising the immediate and long-term results of treatment.

\section{ACKNOWLEDGMENTS}

The authors are grateful to National ENT Hospital for financial support.

\section{CONFLICTS OF INTEREST}

The authors declare no conflict of interest.

\section{REFERENCES}

Baskin RM, Boyce BJ, Amdur R, Mendenhall WM, et al. (2018). Transoral robotic surgery for oropharyngeal cancer: patient selection and special considerations. Cancer Manag. Res. 10: 839-846.

Canis M, Ihler F, Martin A, Matthias C, et al. (2015). Transoral laser microsurgery for T1a glottic cancer: review of 404 cases. Head Neck. 37(6): 889-95.

Chiesa-Estomba CM, González-García JA, Larruscain E, Calvo-Henríquez C, et al. (2019). CO2 Transoral Laser Microsurgery in Benign, Premalignant and Malignant (Tis, T1, T2) Lesion of the Glottis. A Literature Review. Medicines (Base)l. 6(3): 77.

Chiesa-Estomba CM, Reinoso FA, Velasquez AO, Fernandez JL, et al. (2016). Transoral CO2 Laser Microsurgery Outcomes for Early Glottic Carcinomas T1-T2. Int. Arch. Otorhinolaryngol.20(3): 212-217.

Ferlay J, Soerjomataram I, Dikshit R, Eser S, et al. (2015). Cancer incidence and mortality worldwide: sources, methods and major patterns in GLOBOCAN 2012. Int. J. Cancer. 136(5): 359-386.

Forner D, Rigby MH, Corsten M, Trites JR, et al. (2020). Oncological and functional outcomes after repeat transoral laser microsurgery for the treatment of recurrent early glottic cancer. J. Laryngol. Otol. 134(2): 169-173.

Gioacchini FM, Tulli M, Kaleci S, Bondi S, et al. (2017). Therapeutic modalities and oncologic outcomes in the treatment of T1b glottic squamous cell carcinoma: a systematic review. Eur. Arch. Otorhinolaryngol. 274(12): 4091-4102.

Golusinski W and Golusinska-Kardach E (2019). Current Role of Surgery in the Management of Oropharyngeal Cancer. Front. Oncol. 24(9): 388.

Guimarães AV, Dedivitis RA, Matos LL, Aires FT, et al. (2018). Comparison between transoral laser surgery and radiotherapy in the treatment of early glottic cancer: A systematic review and meta-analysis. Sci. Rep. 8(1): 11900 .

Hoffmann C, Cornu N, Hans S, Sadoughi B, et al. (2016). Early glottic cancer involving the anterior commissure treated by transoral laser cordectomy. Laryngoscope. 126(8): 1817-1822.

Howard J, Masterson L, Dwivedi RC, Riffat F, et al. (2016). Minimally invasive surgery versus radiotherapy/chemoradiotherapy for small-volume primary oropharyngeal carcinoma. Cochrane Database Syst. Rev. 12: 010963

Lane C, Rabbani R, Linton J, Taylor SM, et al. (2020). Systematic review and meta-analysis of transoral laser microsurgery in hypopharyngeal carcinoma. Laryngoscope Investig. Otolaryngol. 5(1): 66-73.

Le HJ, Chen SY, Li Y, Xu Y, et al. (2019). The progress on diagnosis and treatment of larynx cancer. Lin Chung Er Bi Yan Hou Tou Jing Wai Ke Za Zhi. 33(11): 1017-1021.

Lei L, Zhong D, Zou J, Wang H, et al. (2019). Oncological outcomes of early stage glottic squamous cell carcinoma treated with transoral laser microsurgery. Am. J. Otolaryngol. 2019: 102364.

Mehanna H, Kong A and Ahmed SK (2016). Recurrent head and neck cancer: United Kingdom National Multidisciplinary Guidelines. J. Laryngol. Otol. 130(S2): 181-190.

Mendelsohn AH and Remacle MJ (2018). Vocal Fold Cancer Transoral Laser Microsurgery Following European Laryngological Society Laser Cordectomy Classification. Front. Oncol. (8): 231.

Mendenhall WM, Werning JW, Hinerman RW, Amdur RJ, Villaret DB (2004). Management of T1-T2 glottic carcinomas. Cancer. 100(9): 1786-1792. doi:10.1002/cncr.20181.

Meulemans J, Delaere P, Nuyts S, Clement PM, et al. (2017). Salvage Transoral Laser Microsurgery for Radiorecurrent Laryngeal Cancer: Indications, Limits, and Outcomes. Curr. Otorhinolaryngol. Rep. 5(1): 83-91. 
Nguyen T and Tran T (2017). Transoral Laser Microsurgery for Early Glottic Cancer. Biomed. Res. Ther. 4(S): S54.

Park JJ and Won S (2019). Voice Outcomes After Transoral Laser Microsurgery or Radiotherapy in Early Glottic Cancer: Factors to Consider. Clin. Exp. Otorhinolaryngol. 12(3): 233-234. doi:10.21053/ceo.2019.00787.

Peretti G, Piazza C, Mora F, Garofolo S, et al. (2016). Reasonable limits for transoral laser microsurgery in laryngeal cancer. Curr. Opin. Otolaryngol. Head Neck Surg. 24(2): 135-139.

Shiner Y, Lubianiker B, and Doweck I (2020). Early laryngeal cancer - treatment outcomes of transoral laser microsurgery. Harefuah. 159(1): 77-82.

Sjögren EV (2017). Transoral Laser Microsurgery in Early Glottic Lesions. Curr. Otorhinolaryngol. Rep. 5(1): 56-68.

Strieth S, Hagemann J and Hess M (2020). Angiolytic laser applications for the larynx: Phonosurgical concepts for transoral laser microsurgery. HNO. 68(1): 59-68.

Tran T and Nguyen T (2018). Early Outcomes of Transoral Laser Microsurgery for T1 - T2 Oropharyngeal Cancer. Asian J. Pharmaceut. Res. Health Care. 10(4): 104-108.

Valls-Mateus M, Ortega A Blanch JL, Sabater F, et al. (2016). Long-term quality of life after transoral laser microsurgery for laryngeal carcinoma. Surg. Oncol. 114(7): 789-795.

Vilaseca I, Bernal-Sprekelsen M, Him R, Mandry A, et al. (2015). Prognostic factors of quality of life after transoral laser microsurgery for laryngeal cancer. Eur. Arch. Otorhinolaryngol. 272(5): 1203-1210.

Warner L, Lee K and Homer JJ (2017). Transoral laser microsurgery versus radiotherapy for T2 glottic squamous cell carcinoma: a systematic review of local control outcomes. Clin. Otolaryngol. 42(3): 629-636.

Wiegand S (2016). Evidence and evidence gaps of laryngeal cancer surgery. GMS Curr. Top Otorhinolaryngol. Surg. 15: Doc03. 